\title{
Hesperidin ameliorates liver ischemia/reperfusion injury via activation of the Akt pathway
}

\author{
SHILAI LI ${ }^{1}$, QUANLIN QIN ${ }^{1}$, DAQING LUO ${ }^{1}$, WENHUI PAN ${ }^{1}$, YUQING WEI ${ }^{1}$, \\ YANSONG XU ${ }^{1}$, JIJIN ZHU ${ }^{1}$ and LIMING SHANG ${ }^{2}$ \\ Departments of ${ }^{1}$ Emergency and ${ }^{2}$ Hepatobiliary Surgery, The First Affiliated Hospital of \\ Guangxi Medical University, Nanning, Guangxi 530021, P.R China
}

Received March 22, 2020; Accepted July 3, 2020

DOI: $10.3892 / \mathrm{mmr} .2020 .11561$

\begin{abstract}
Hesperidin (HDN) is a bioflavonoid that serves a role as an antioxidant in biological systems. However, although HDN has hydrogen radical- and hydrogen peroxide-removal activities, the role of HDN in liver ischemia/reperfusion (I/R) injury remains unknown. This study aimed to determine the role of HDN in liver I/R injury. Male C57BL/6J wild-type (WT) mice were subjected to warm partial liver I/R injury. Liver damage was evaluated by measuring serum alanine aminotransferase (ALT) levels, cytokine production, oxidative stress indicators, tissue hematoxylin-eosin staining and cell death. The Akt signaling pathway was examined to elucidate the underlying mechanisms. HDN had no effect on ALT levels and tissue damage in WT mice without liver I/R injury. However, HDN significantly ameliorated liver I/R injury as measured by serum ALT levels and necrotic tissue areas. HDN decreased malondialdehyde content, but increased the levels of superoxide dismutase, catalase, glutathione peroxidase and glutathione. In addition, HDN significantly attenuated the mRNA expression levels of TNF- $\alpha$, IL- 6 and IL- $1 \beta$ after liver I/R injury. Furthermore, HDN protected the liver against apoptosis in liver I/R injury by increasing the levels of Bcl-2 and decreasing the levels of cleaved-caspase 3. Mechanistically, the levels of phosphorylated Akt were elevated by HDN during liver I/R injury. In addition, HDN could induce Akt activation in hepatocytes in vitro. Most importantly, treatment with the Akt inhibitor LY294002 in WT mice blocked the hepatoprotective effects of HDN in liver I/R injury. In summary, the
\end{abstract}

Correspondence to: Dr Jijin Zhu, Department of Emergency, The First Affiliated Hospital of Guangxi Medical University, 6 Shuangyong Road, Nanning, Guangxi 530021, P.R. China

E-mail: zhujijin63@vip.sina.com

Dr Liming Shang, Department of Hepatobiliary Surgery, The First Affiliated Hospital of Guangxi Medical University, 6 Shuangyong Road, Nanning, Guangxi 530021, P.R. China

E-mail: shangliming@gxmu.edu.cn

Key words: hesperidin, liver ischemia/reperfusion injury, oxidative stress, apoptosis, Ak results of the present study suggested that HDN may protect against liver I/R injury through activating the Akt pathway by ameliorating liver oxidative stress, suppressing inflammation and preventing hepatocyte apoptosis. HDN may be a useful factor for liver injury protection and a potential therapeutic treatment for liver $\mathrm{I} / \mathrm{R}$ injury in the future.

\section{Introduction}

Ischemia-reperfusion (IR) injury remains a key medical challenge because it relates to organ transplantation and alterations in vascular perfusion (1-3). Liver I/R injury frequently occurs as a result of liver transplantation, partial hepatectomy, hemorrhagic shock, trauma, severe infection and metabolic disorder $(1,4)$. I/R injury contributes to $10 \%$ of early graft dysfunction following organ transplantation and accounts for acute and chronic rejection $(5,6)$. Much effort has been made to elucidate the underlying mechanisms of $I / R$ injury, but so far little is understood $(7,8)$. Oxidative stress, innate and adaptive immune responses, excessive inflammation, anaerobic metabolism and acidosis are considered to be the main molecular and cellular events that contribute to liver injury following I/R (7-9). However, no approved clinical intervention is currently available for I/R-related liver injury (10). Our latest study indicated that thymic stromal lymphopoietin protects against liver I/R injury via activation of the PI3K/Akt pathway (11). Akt is thought to be a potential therapeutic target in liver I/R injury (12). Thus, seeking factors that can activate Akt pathway may be a promising protection strategy for liver $\mathrm{I} / \mathrm{R}$ injury.

Hesperidin (HDN) is a bioflavonoid, abundant in citrus fruits, such as oranges, grapefruits and lemons, that serves a role as an antioxidant in biological systems (13). It has been confirmed that HDN has hydrogen radical- and hydrogen peroxide-removal activities $(13,14)$. Additionally, it has been reported that HDN has numerous beneficial effects, such as anti-inflammatory, antibacterial, anticancer and anti-edema properties (13-16). Thus, HDN has a protective effect on sterile organ injuries, including ischemic stroke and arthritis, and infectious diseases such as lipopolysaccharide-induced inflammation (17-20). A number of studies reveal that the activation of PI3K/Akt pathway is one of the most important mechanisms for the biological effects of $\operatorname{HDN}(16,18,21,22)$. 
Recently, Park et al (23) observed that HDN ameliorates hepatic injury in Sprague-Dawley rats. However, the underlying mechanisms remain unknown.

The present study hypothesized that HDN ameliorates liver I/R injury through the Akt pathway. Wild-type (WT) mice were subjected to liver I/R and administered HDN. HDN ameliorated liver I/R injury, but the hepatoprotective effects of HDN were prevented by an Akt inhibitor. The results of the present study demonstrated that HDN ameliorated liver oxidative stress, suppressed inflammatory responses and prevented hepatocyte apoptosis during liver I/R injury. Thus, HDN administration may be useful to prevent injury after liver I/R.

\section{Materials and methods}

Animals and model. A total of 65 male C57BL/6J WT mice (8-10 weeks old, 22-30 g) were purchased and housed in the Guangxi Medical University Laboratory Animal Center (Guangxi, China). The mice were bred in a specific pathogen-free animal facility under controlled conditions at $19-23^{\circ} \mathrm{C}$ and $40-60 \%$ humidity with a 12 -h dark/light cycle and had free access to food and water. A warm partial liver I/R injury model with occlusion of $70 \%$ segments of the liver blood supply was established as previously described (24). Briefly, the mice were injected intraperitoneally (i.p.) with ketamine $(100 \mathrm{mg} / \mathrm{kg})$-xylazine $(5 \mathrm{mg} / \mathrm{kg})$. The left and middle hepatic lobe blood supply was occluded with an atraumatic clamp (Fine Science Tools, Inc.). The clamp was removed after $60 \mathrm{~min}$ of ischemia, and the reperfusion procedure lasted for $6 \mathrm{~h}$. HDN was administered orally to the mice by gavage for 3 days before surgery. To ascertain the optimal dose for the 3-day HDN administration, a fixed-dose study was designed with doses of 100, 200 and $400 \mathrm{mg} / \mathrm{kg}$. LY294002 $(0.5 \mathrm{mg} / \mathrm{kg}$, i.p.) was administered $30 \mathrm{~min}$ before surgery. Sham (treated without HDN) and control (treated with HDN at the dose of $200 \mathrm{mg} / \mathrm{kg}$ for 3 days) mice underwent anesthesia, laparotomy and exposure of the portal triad but without vasculature occlusion. Mice were euthanized by exsanguination under anesthesia following reperfusion for $6 \mathrm{~h}$. Mice were anaesthetized with $1.5 \%$ isoflurane, $1 \mathrm{ml}$ of blood was harvested by cardiac puncture and liver samples were collected and stored as detailed below. Following blood and tissue collection, euthanasia was confirmed by bilateral thoracotomy and subsequently by the absence of any respiratory movement and heartbeat.

The whole blood of the mice was collected in a covered test tube and allowed to clot by leaving it undisturbed for $20 \mathrm{~min}$ at room temperature. The clot was removed by centrifuging at $2,000 \mathrm{x} \mathrm{g}$ for $10 \mathrm{~min}$ in a refrigerated $\left(4^{\circ} \mathrm{C}\right)$ centrifuge. The resulting supernatant was designated serum and was immediately transferred into a clean polypropylene tube using a pipette. The serum was stored at $-80^{\circ} \mathrm{C}$ for further analysis. Part of the liver samples were embedded in $2 \%$ paraformaldehyde for histological analysis, and the remainder were frozen in liquid nitrogen and stored at $-80^{\circ} \mathrm{C}$ for further analysis.

The mice were divided into the following groups: i) Sham group ( $\mathrm{n}=8)$; ii) control group $(\mathrm{n}=8)$; iii) $\mathrm{I} / \mathrm{R}$ group $(\mathrm{n}=8)$; iv) $\mathrm{I} / \mathrm{R}+\mathrm{HDN}$ group ( $\mathrm{n}=8 /$ group, at three different concentrations: 100, 200 and $400 \mathrm{mg} / \mathrm{kg}$ ); v) I/R+LY294002 group $(\mathrm{n}=8)$; and vi) $\mathrm{I} / \mathrm{R}+\mathrm{HDN}(200 \mathrm{mg} / \mathrm{kg})+\mathrm{LY} 294002$ group $(\mathrm{n}=8)$.
Reagents. Antibodies for western blotting were as follows: the GAPDH antibody (cat. no. ab8245) was purchased from Abcam, and the Bcl-2 (cat. no. 3498), pro-caspase 3 (cat. no. 9662), cleaved caspase 3 (cat. no. 9661), phosphorylated (p)-Akt (S473) (cat. no. 9271) and Akt antibodies (cat. no. 9272) were purchased from Cell Signaling Technology, Inc. Goat anti-mouse secondary antibody (cat. no. 31430) and goat anti-rabbit secondary antibody (cat. no. 31460, both at 1:20,000 dilution) were from Thermo Fisher Scientific, Inc. HDN (HPLC $>98 \%$; cat. no. XW05202631) was obtained from Sinopharm Chemical Reagent Co., Ltd. LY294002 (cat. no. L9908) was purchased from Sigma-Aldrich (Merck KGaA). Malondialdehyde assay kit (MDA; cat. no. A003-1), superoxide dismutase assay kit (SOD; cat. no. A001-1), catalase assay kit (CAT; cat. no. A007-2), glutathione peroxidase assay kit (GPx; cat. no. A006-1) and glutathione assay kit (GSH; cat. no. A006-1) were purchased from Nanjing Jiancheng Bioengineering Institute.

Hepatocyte isolation and culture. Hepatocytes (HCs) were isolated as described previously (25). Mice were euthanized with 5\% isoflurane for 5 minutes in a plexiglass chamber, and bilateral thoracotomy was performed for a secondary confirmation of death. The mice were perfused following euthanasia in vivo by using an in situ collagenase (type VI, Worthington Biochemical Corporation) technique. Next, HCs were separated from nonparenchymal cells (NPCs) by two cycles of differential centrifugation $\left(50 \mathrm{x}\right.$ g for $2 \mathrm{~min}, 4^{\circ} \mathrm{C}$ ) to ensure that the purification exceeded 99\%. Finally, trypan blue exclusion was used to test the viability to ensure that it was greater than $95 \%$. HCs were cultured as described previously (26). Briefly, hepatocytes $(1.5 \times 105$ cells $/ \mathrm{ml})$ were plated on gelatin-coated culture plates with collagen I (BD Pharmingen; BD Biosciences) in Williams medium E (Invitrogen; Thermo Fisher Scientific, Inc.) with $10 \%$ calf serum (Thermo Fisher Scientific, Inc.), 15 mM HEPES (Thermo Fisher Scientific, Inc.), $1 \mu \mathrm{M}$ insulin (Eli Lilly and Company), $2 \mathrm{mML}$-glutamine (Thermo Fisher Scientific, Inc.), penicillin (100 U/ml) and streptomycin (100 U/ml; Invitrogen; Thermo Fisher Scientific, Inc.) at $37^{\circ} \mathrm{C}$. Cells were allowed to attach to plates overnight, and the culture media was replaced with fresh culture media before the cells were treated for experiments.

Immunofluorescence staining. After hepatocytes were cultured for $24 \mathrm{~h}$ at $37^{\circ} \mathrm{C}$, cells were washed by phosphate-buffered saline (PBS; pH 7.4) once, and then were fixed with $4 \%$ paraformaldehyde (Rich Joint) for $30 \mathrm{~min}$ at $4^{\circ} \mathrm{C}$. Cells were then permeabilized by $0.1 \%$ Triton-X 100 (Rich Joint) for $15 \mathrm{~min}$ at room temperature, washed twice with PBS, incubated with $5 \%$ fetal bovine serum (HyClone; Thermo Fisher Scientific, Inc.) for $15 \mathrm{~min}$ at room temperature and then incubated with hepatocyte-specific marker Cytokeratin-18 (CK18) primary antibody (1:1,000 dilution) (cat. no. 10830-1-AP; Wuhan Sanying Biotechnology) overnight at $4^{\circ} \mathrm{C}$. Cells were then washed with PBS three times (5 min each) and incubated with fluorescein isothiocyanate (FITC)-conjugated goat anti-rabbit secondary antibody (1:100 dilution; cat. no. SA00003-2; Wuhan Sanying Biotechnology) at $37^{\circ} \mathrm{C}$ for $1 \mathrm{~h}$, followed by Hoechst 33258 (cat. no. C1017; Beyotime Institute of Biotechnology) for nuclear staining for $15 \mathrm{~min}$ at room temperature. Slides 
A

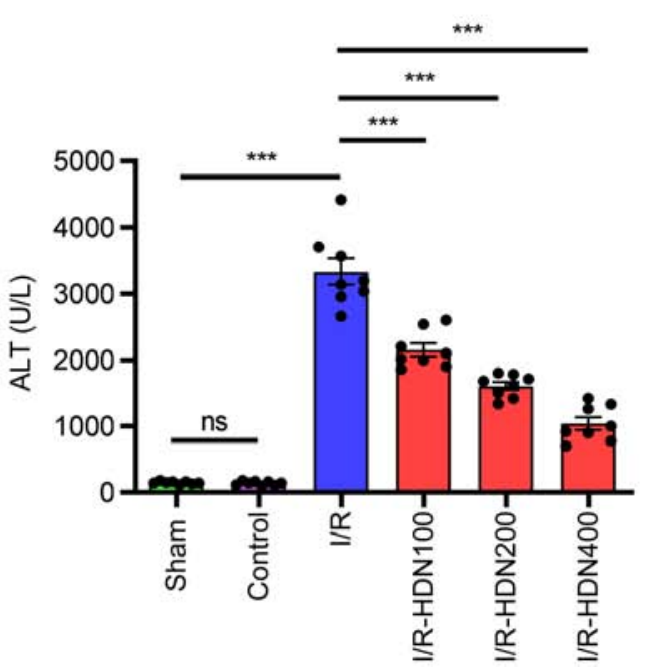

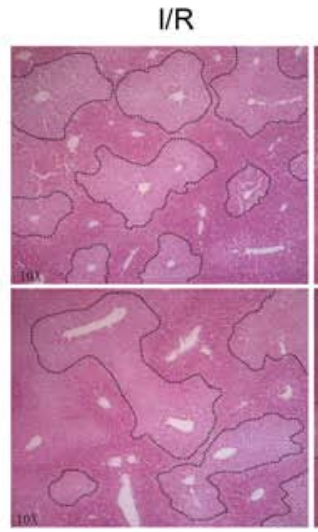

I/R-HDN100

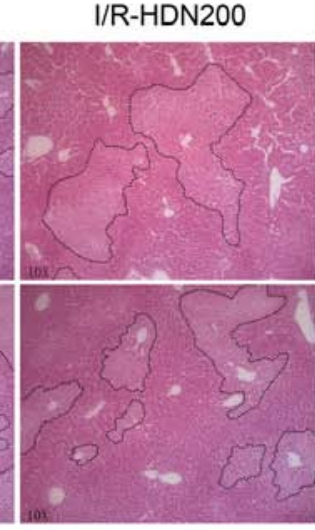

I/R-HDN400

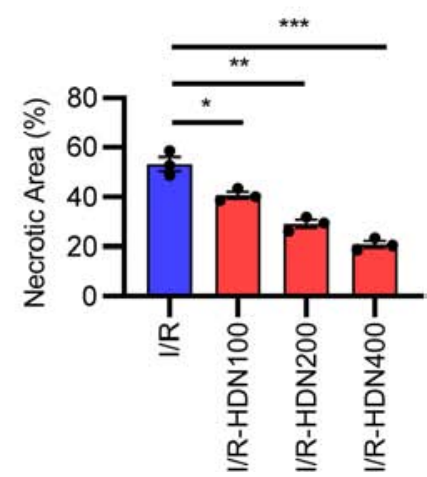

Figure 1. HDN ameliorates liver I/R injury. (A) Serum ALT levels in the livers of mice after sham surgery, control, I/R injury or I/R injury with 100, 200 or $400 \mathrm{mg} / \mathrm{kg}$ HDN treatment; $\mathrm{n}=8$ mice/group. (B) Representative hematoxylin and eosin staining images and necrotic areas of ischemic liver lobes of mice at $6 \mathrm{~h}$ post-reperfusion with or without HDN treatment; magnification, $\mathrm{x} 10$. Dotted lines indicate measured areas of necrosis, quantified on bar graph. $\mathrm{n}=8 \mathrm{mice} /$ group. ${ }^{*} \mathrm{P}<0.05,{ }^{* *} \mathrm{P}<0.01$ and ${ }^{* * *} \mathrm{P}<0.001$. ALT, alanine aminotransferase; HDN, hesperidin; $\mathrm{I} / \mathrm{R}$, ischemia/reperfusion; ns, not significant.
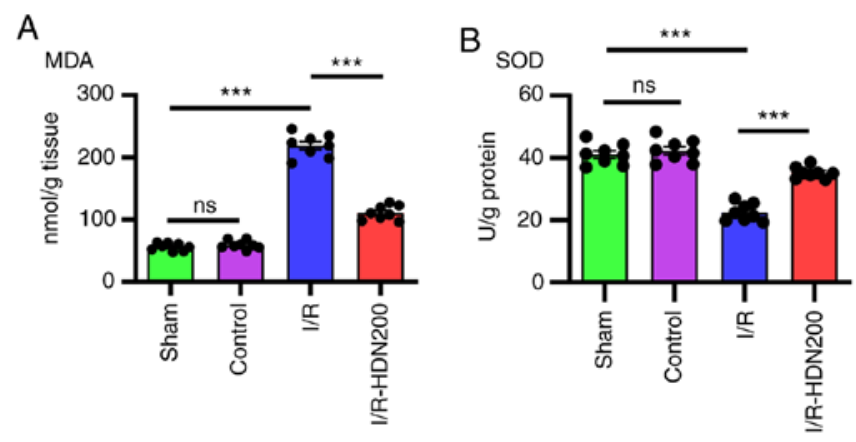

C

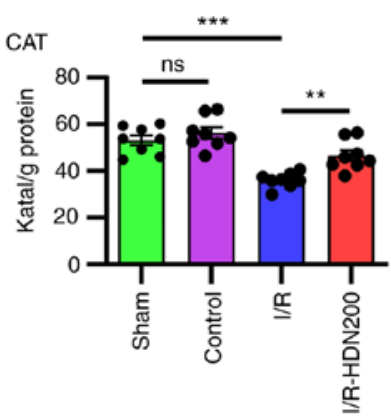

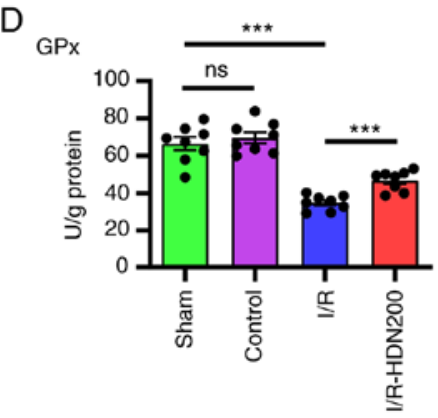

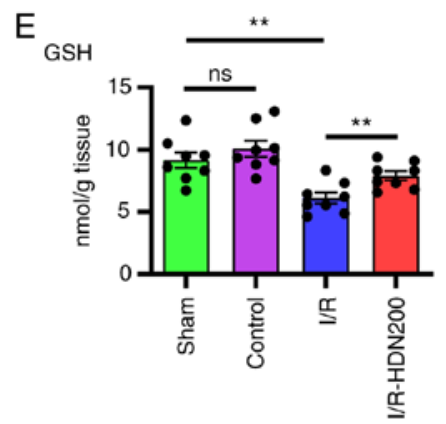

Figure 2. HDN ameliorates liver oxidative stress in liver I/R injury. The levels of (A) MDA, (B) SOD, (C) CAT, (D) GPx and (E) GSH of liver lobes in sham, control, I/R or I/R with HDN treatment $(200 \mathrm{mg} / \mathrm{kg})$ groups. $\mathrm{n}=8$ mice/group; ${ }^{* *} \mathrm{P}<0.01$ and ${ }^{* * * *} \mathrm{P}<0.001$. CAT, catalase; GPx, glutathione peroxidase; GSH, glutathione; HDN, hesperidin; I/R, ischemia/reperfusion; MDA, malondialdehyde; ns, not significant; SOD, superoxide dismutase.

were viewed using an Olympus IX71 fluorescence microscope (Olympus Corporation).

Serum sample assays. Serum alanine aminotransferase (ALT) levels were measured by alanine aminotransferase assay kit using standard spectrophotometric procedures with $5 \mu 1$ serum according to the manufacturer's instructions (cat. no. C009-2-1, Nanjing Jiancheng Institute of Biotechnology). The levels of ALT were expressed as units/L serum (U/L).
Histological analysis. Liver tissues were harvested as described in the Animals and model subsection, embedded in $2 \%$ paraformaldehyde for $2 \mathrm{~h}$ at $4^{\circ} \mathrm{C}$ and then removed for preservation at $4^{\circ} \mathrm{C}$ with $70 \%$ ethanol. Liver sections $(5 \mu \mathrm{m})$ were stained with hematoxylin and eosin $(\mathrm{H} \& \mathrm{E})$ to assess histopathology. Images of five randomly selected fields were acquired with a light microscope (magnification, $\mathrm{x} 10$ ). Necrotic areas were analyzed by ImageJ software (version 1.51w, National Institutes of Health). 
A

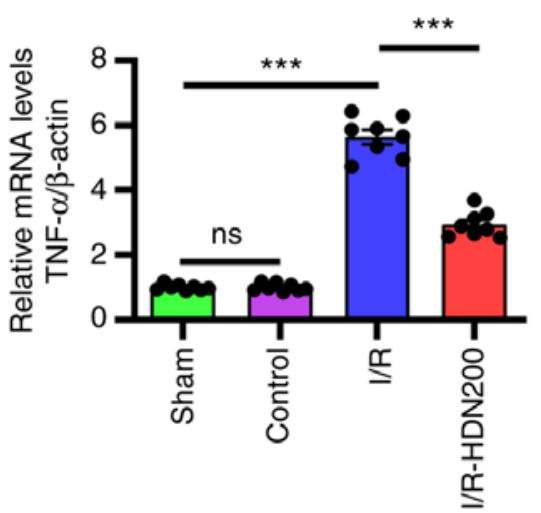

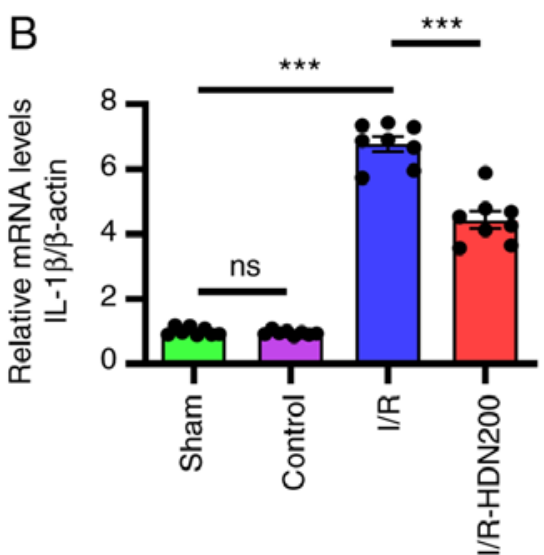

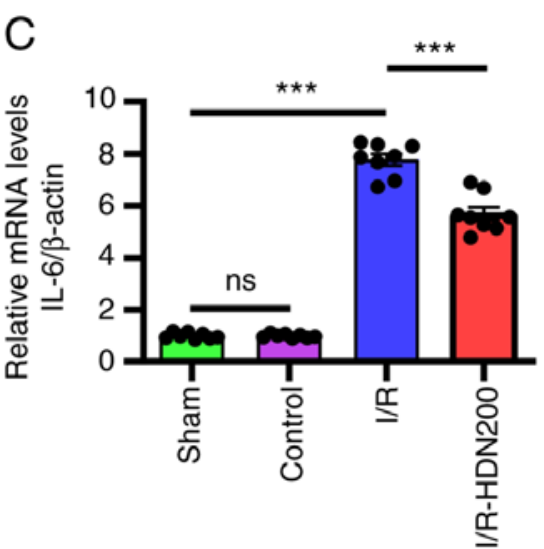

Figure 3. HDN suppresses inflammation during liver I/R injury. mRNA expression levels of (A) TNF- $\alpha$, (B) IL-1 $\beta$ and (C) IL-6 assessed by reverse transcription-quantitative PCR in liver tissues from mice in sham, control, I/R or I/R with HDN treatment (200 mg/kg) groups; $\beta$-Actin served as a control. $\mathrm{n}=8 \mathrm{mice} /$ group. Results are expressed as the relative fold increase from three experiments. ${ }^{* * *} \mathrm{P}<0.001$. HDN, hesperidin; I/R, ischemia/reperfusion; ns, not significant.

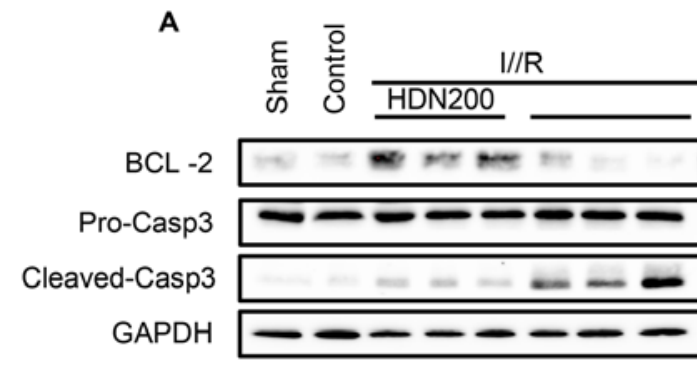

B

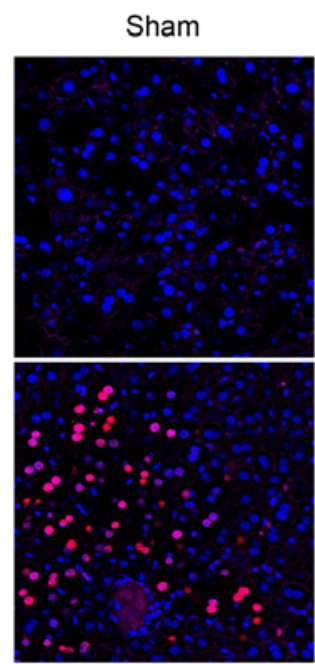

I/R
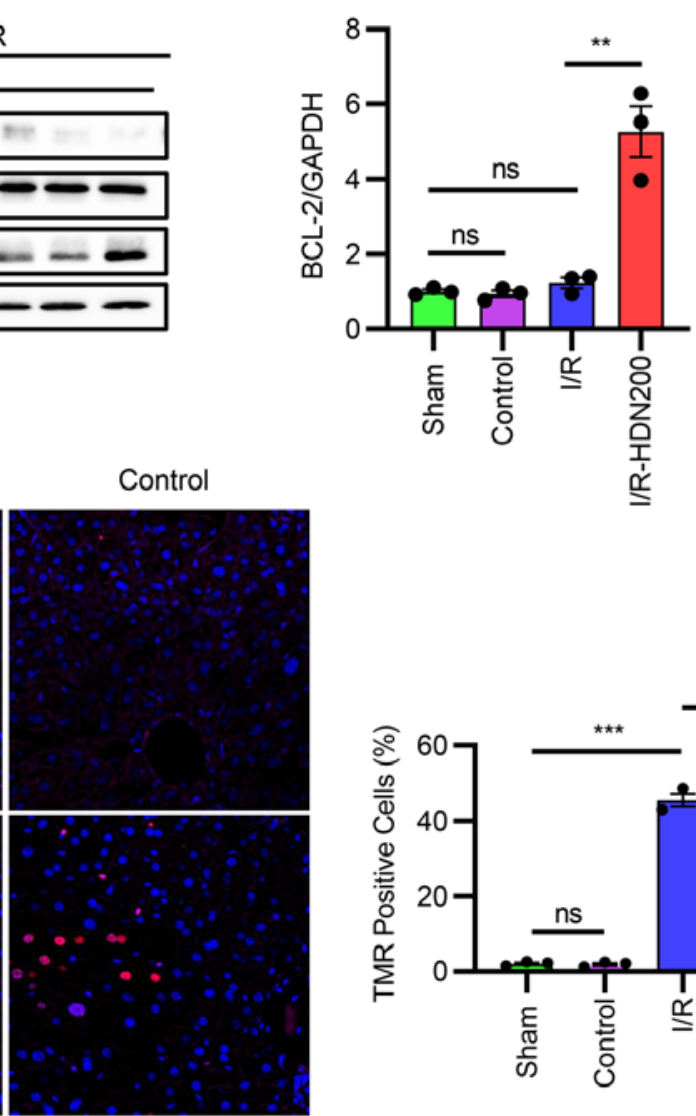

I/R-HDN200

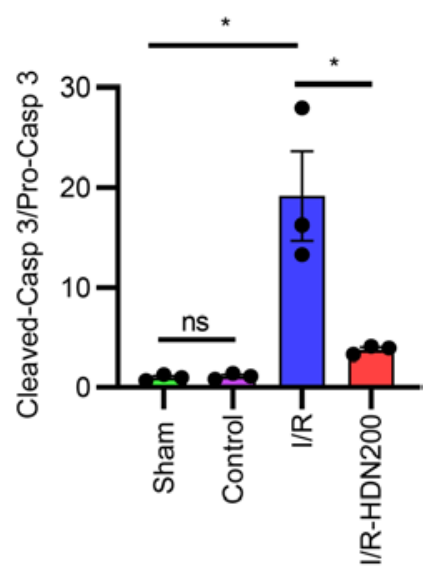

Figure 4. HDN protects the liver against apoptosis in liver I/R injury. (A) Western blotting analysis of Bcl-2 and cleaved-casp3 protein expression in the liver of mice after I/R injury with or without HDN treatment. (B) Confocal images from mouse livers stained with TMR red (red) and Hoechst nuclear stain (blue) after sham surgery or liver I/R injury with or without HDN (200 mg/kg) treatment. Magnification, x40. Percentage of TMR-positive cells was quantified using ImageJ software and represented in a bar graph. $\mathrm{n}=8$ mice/group; ${ }^{*} \mathrm{P}<0.05,{ }^{* *} \mathrm{P}<0.01$ and ${ }^{* * *} \mathrm{P}<0.001$. Casp3, caspase 3 ; HDN, hesperidin; I/R, ischemia/ reperfusion.; ns, not significant.

Reverse transcription-quantitative PCR (RT-qPCR). The ischemic lobe of the liver was frozen in liquid nitrogen and stored at $-80^{\circ} \mathrm{C}$ for comparative PCR analysis. Total RNA was extracted with the RNeasy Mini Kit (Qiagen China Co., Ltd.) according to the manufacturer's instructions. cDNA was synthesized using $1 \mu \mathrm{g}$ of RNA and with $2 \mu \mathrm{M}$ oligodT primers (Qiagen China Co., Ltd.), 4 U Omniscript ${ }^{\mathrm{TM}}$ reverse transcriptase (Qiagen China Co., Ltd.), $4 \mu \mathrm{l}$ of 5X RT buffer, and $16 \mu \mathrm{l}$ RNA in RNase-free water. The synthesis of cDNA was performed at $37^{\circ} \mathrm{C}$ for $60 \mathrm{~min}$. SYBR Green PCR Master Mix 

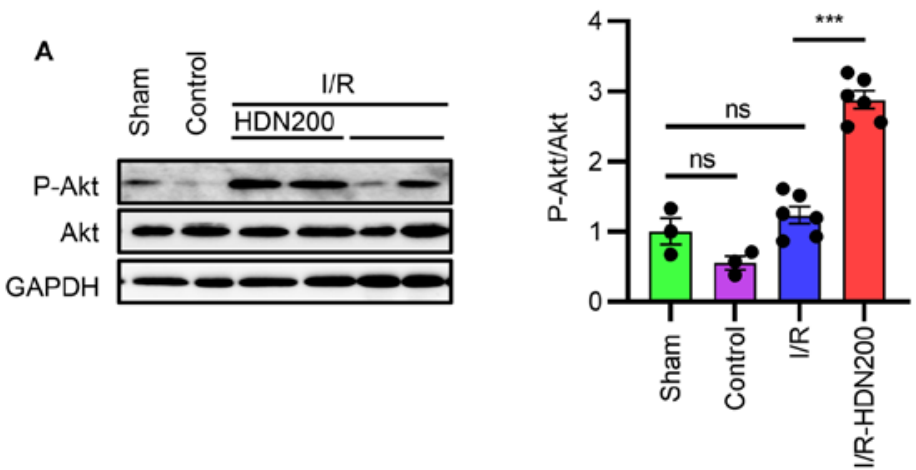

B
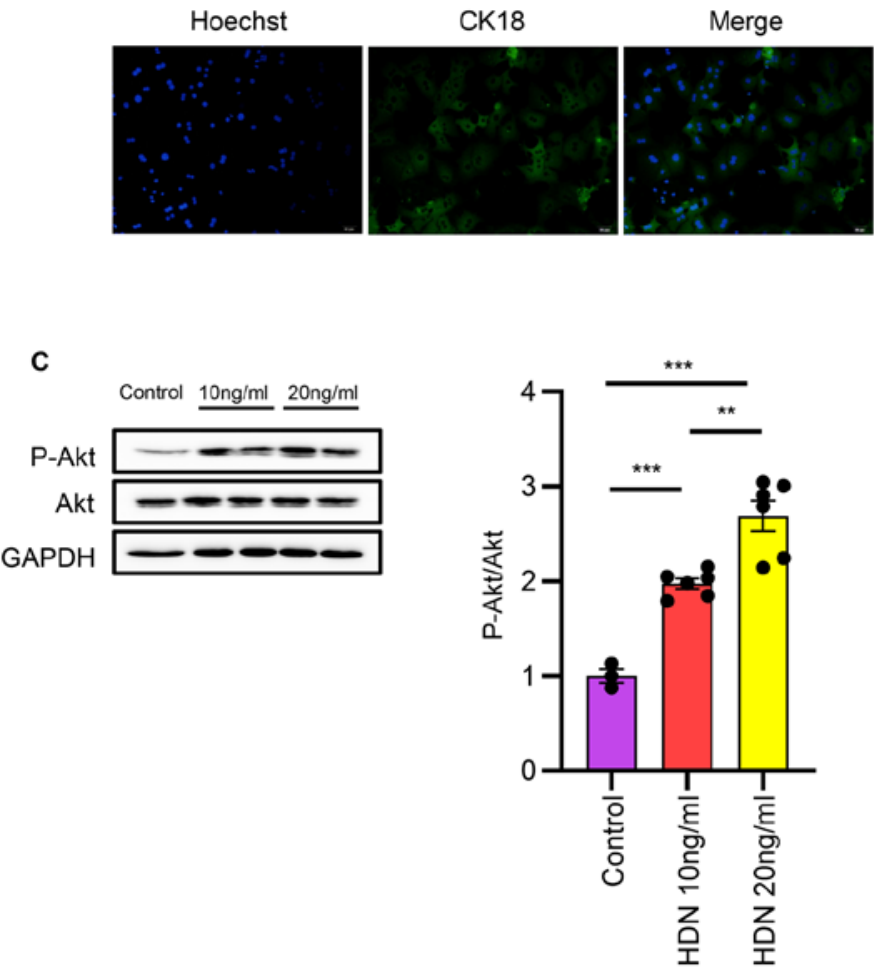

Figure 5. HDN activates the Akt pathway in liver I/R injury in vivo and in hepatocytes in vitro. (A) Western blotting showing the levels of total and p-Akt in liver of mice after liver I/R injury with or without HDN treatment. Each lane represents a separate animal. (B) Immunofluorescence staining showing Hoechst (blue) and CK18 (green) positive cells. Magnification, x200 (C) Western blotting showing the levels of total and p-Akt in primary hepatocytes after exposed to different doses of HDN $(10$ and $20 \mathrm{ng} / \mathrm{ml} ; 6 \mathrm{~h})$ in vitro. The blots shown are representative of three experiments with similar results. ${ }^{* *} \mathrm{P}<0.01$ and ${ }^{* * *} \mathrm{P}<0.001$. CK18, cytokeratin-18; HDN, hesperidin; I/R, ischemia/reperfusion; p-, phosphorylated; ns, not significant.

(Applied Biosystems; Thermo Fisher Scientific, Inc.) was used to prepare the PCR reaction mixes. qPCR was performed with sense and antisense primer pre-validated and specific for $\beta$-actin, IL-1 $\beta$, IL-6, and TNF- $\alpha$ (Qiagen China Co., Ltd.) with the following thermocycling parameters: Initial denaturation at $94^{\circ} \mathrm{C}$ for $5 \mathrm{~min}$; followed by 40 cycles of $94^{\circ} \mathrm{C}$ for $15 \mathrm{sec}$, $60^{\circ} \mathrm{C}$ for $20 \mathrm{sec}$ and $72^{\circ} \mathrm{C}$ for $30 \mathrm{sec}$. The reaction cocktail used for test contained $10 \mu \mathrm{l}$ iTaq SYBR Green PCR Master Mix, $8 \mu \mathrm{l}$ RNase-free water, $0.5 \mu \mathrm{l}$ sense primer, $0.5 \mu \mathrm{l}$ antisense primer and $1 \mu \mathrm{l} \mathrm{cDNA}$. All samples were assayed in duplicate and normalized to $\beta$-actin mRNA abundance. Gene expression levels were quantified using the $2^{-\Delta \Delta \mathrm{Cq}}$ method (27). The primers used for qPCR were as follows: $\beta$-actin, forward 5'-TGTGATGGTGGGAATGGGTCAG-3', reverse 5'-TTT GATGTCACGCACGATTTCC-3'; IL-1 $\beta$, forward 5'-CCA GCTTCAAATCTCACAGCAG-3', reverse 5'-CTTCTTTGG GTATTGCTTGGGATC-3'; IL-6, forward 5'-TCCAGTTGC
CTTCTTGGGAC-3', reverse 5'-GTACTCCAGAAGACC AGAGG-3'; and TNF- $\alpha$, forward 5'-CACAGAAAGCATGAT CCGCGACGT-3', reverse 5'-CGGCAGAGAGGAGGTTGA CTTTCT-3'.

Western blotting analysis. Protein expression was determined in frozen liver tissues using a previously described western blotting protocol (11). Briefly, snap-frozen liver was homogenized in cell lysis buffer (Cell Signaling Technology, Inc.) and centrifuged $(16,000 \mathrm{x} g$ for $15 \mathrm{~min}$, $4^{\circ} \mathrm{C}$ ), after which the supernatant was collected. Protein concentrations were determined using a BCA Protein Assay Kit (Thermo Fisher Scientific, Inc.). Loading buffer was added to the samples (30 $\mu \mathrm{g} /$ well), which were then resolved by 10 or $15 \%$ SDS-PAGE. Samples were then transferred to a polyvinylidene difluoride membrane at $250 \mathrm{~mA}$ for $2 \mathrm{~h}$. The membrane was blocked in 5\% milk for 


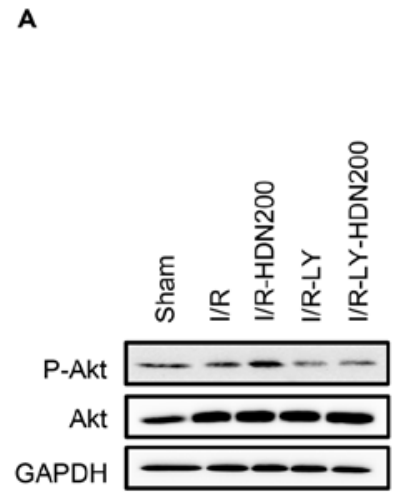

C

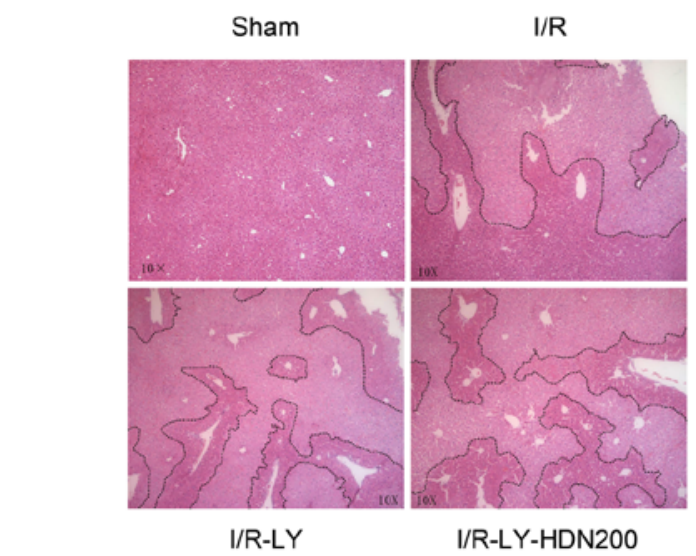

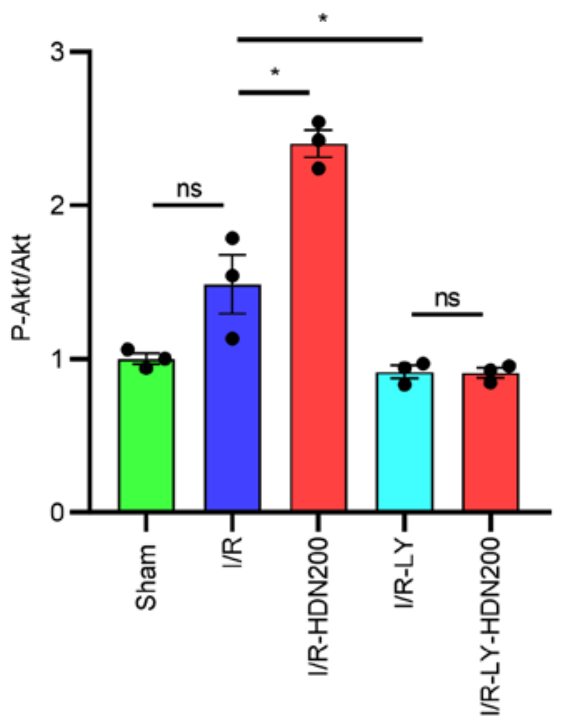

B

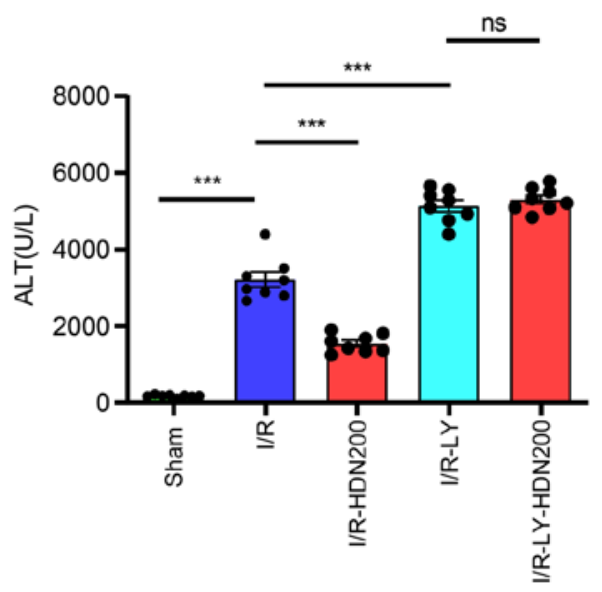

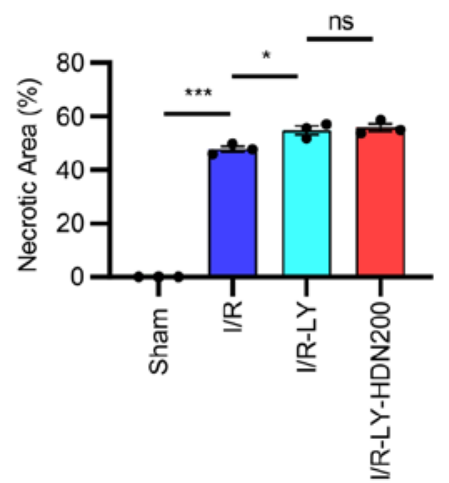

Figure 6. HDN ameliorates liver I/R injury via the Akt signaling pathway. (A) Western blotting showing the levels of total and p-Akt in liver of mice after I/R injury with HDN (200 mg/kg) or Akt inhibitor (LY294002) or LY294002 plus HDN treatment. Each lane represents a separate animal. The blots shown are representative of three experiments with similar results. (B) Serum ALT levels of mice after liver I/R injury treated with HDN (200 mg/kg), LY294002 or LY294002 plus HDN treatment. (C) Representative hematoxylin and eosin staining images and necrotic areas of ischemic liver lobes of mice at $6 \mathrm{~h}$ post-reperfusion with LY294002 or LY294002 plus HDN treatment; dotted lines indicate measured areas of necrosis, quantified on bar graph. Magnification, $\mathrm{x} 10$. $\mathrm{n}=8$ mice/group; ${ }^{*} \mathrm{P}<0.05$ and ${ }^{* * *} \mathrm{P}<0.001$. ALT, alanine aminotransferase; HDN, hesperidin; I/R, ischemia/reperfusion; LY, LY294002; ns, not significant; p-, phosphorylated.

$1 \mathrm{~h}$ at room temperature and then incubated with primary antibody (1:1,000 dilution) in $1 \%$ milk overnight at $4^{\circ} \mathrm{C}$. Membranes were washed in Tris-buffered saline- $0.05 \%$ Tween-20 (TBST) for $10 \mathrm{~min}$, incubated with horseradish peroxidase-conjugated secondary antibody $(1: 20,000$ dilution) for $1 \mathrm{~h}$ and then washed for $1 \mathrm{~h}$ in TBST at room temperature, before being developed by an enhanced chemiluminescence kit (Thermo Fisher Scientific Inc.). The signal was acquired and quantified with a ChemiDoc ${ }^{\mathrm{TM}} \mathrm{MP}$ Imaging System (Bio-Rad Laboratories, Inc.).

Cell death detection assay. Cell death was detected as described previously (25). Briefly, liver tissues were incubated with the In-Situ Cell Death Detection TMR Red kit (cat. no. 12156792910; Roche Diagnostics) according to the manufacturer's instructions. Images (magnification, x40) were captured with a Nikon A1 confocal microscope (Nikon Instruments) from at least six randomly selected fields/sections. Dead cells were quantified by NIS Elements (version 4.1, Nikon Instruments).
Statistical analysis. All experiments were performed in triplicate. All data are presented as the mean \pm standard error of the mean. Two-tailed unpaired Student's t-test was performed to compare the differences between two experimental groups. One-way ANOVA followed by a Bonferroni post hoc test was performed to compare the differences between multiple groups. GraphPad Prism (version 8.0.2; GraphPad Software, Inc.) was used to perform the data analysis. $\mathrm{P}<0.05$ was considered to indicate a statistically significant difference.

\section{Results}

Hesperidin ameliorates liver $I / R$ injury. To determine the role of HDN in liver I/R, WT mice were subjected to warm partial liver I/R injury with or without HDN treatment. Liver injury was assessed by detecting serum ALT levels and morphological indexes (H\&E staining). Compared with that of the sham group the ALT levels indicated that liver damage was markedly increased after liver I/R (Fig. 1A). There was 
A

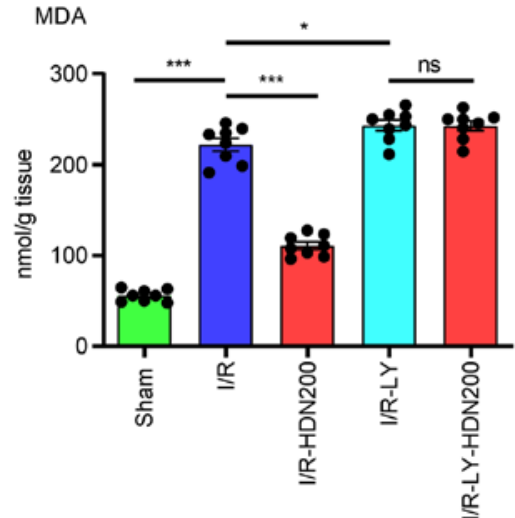

C CAT

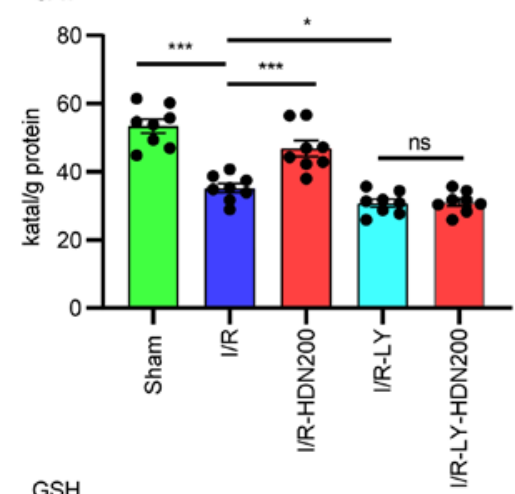

E GSH

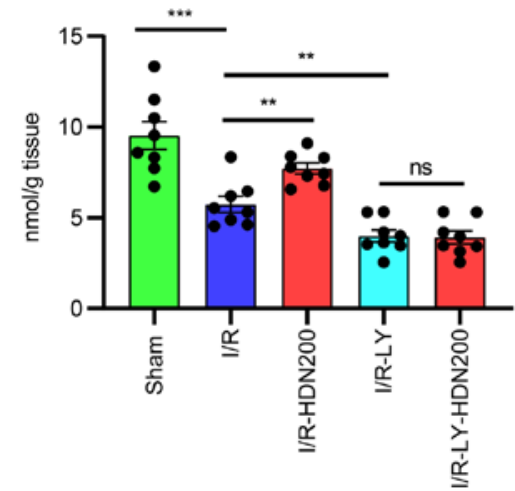

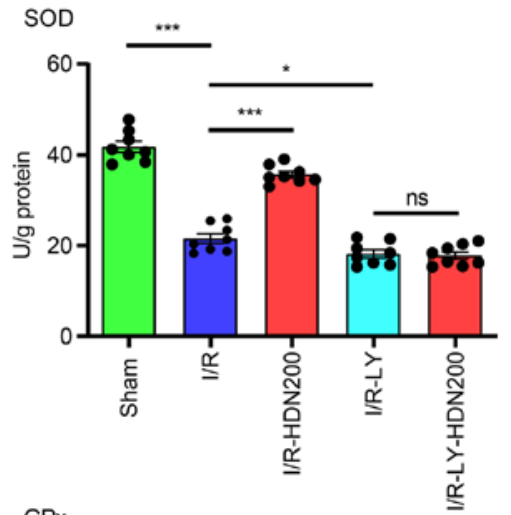

D $\quad \mathrm{GPx}$

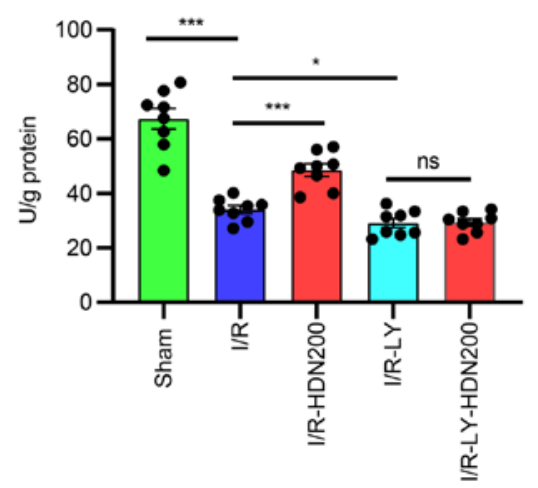

Figure 7. HDN ameliorates liver oxidative stress during I/R injury through the Akt signaling pathway. The levels of (A) MDA, (B) SOD, (C) CAT, (D) GPx and (E) GSH of livers in sham, I/R, I/R with HDN treatment (200 mg/kg), I/R with Akt inhibitor (LY294002) treatment or I/R with LY294002 plus HDN treatment groups. $\mathrm{n}=8$ mice/group; ${ }^{*} \mathrm{P}<0.05,{ }^{* *} \mathrm{P}<0.01$ and ${ }^{* * * *} \mathrm{P}<0.001$. CAT, catalase; GPx, glutathione peroxidase; GSH, glutathione; HDN, hesperidin; I/R, ischemia/ reperfusion; LY, LY294002; MDA, malondialdehyde; ns, not significant; SOD, superoxide dismutase.

no effect on ALT levels in control group but without liver I/R (Fig. 1A). However, HDN significantly ameliorated liver I/R injury in a dose-dependent way (Fig. 1A). H\&E staining also indicated that the necrotic areas of the ischemic hepatic lobe were significantly attenuated by HDN treatment (Fig. 1B). In order to explore the underlying mechanisms, the median concentration of HDN (200 mg/kg) was used for the following experiments.

Hesperidin ameliorates liver oxidative stress in liver $I / R$ injury. Oxidative stress is considered to serve a key role in liver I/R injury $(9,28)$. It has been reported that HDN eliminates oxidative stress in a number of diseases, such as toxin-induced tissue damage and inflammation $(13,14)$. To ascertain whether HDN ameliorates oxidative stress during liver I/R, the levels of MDA, SOD, CAT, GPx and GSH in ischemic liver lobes were measured using commercial kits. Notably, compared with that of the sham group MDA content increased significantly after liver I/R, and this was significantly reversed by HDN treatment (Fig. 2A). In addition, compared with that of the sham group the antioxidant activities of SOD, CAT, GPx and GSH, decreased significantly after liver $I / R$, and these effects were reversed by HDN treatment (Fig. 2B-E).

Hesperidin suppresses inflammation during liver $I / R$ injury. Inflammation serves important roles in liver $I / R$ injury, and numerous cytokines are involved in liver injury $(7,29)$. To determine the relationship between HDN and inflammation during liver I/R injury, the mRNA levels of TNF- $\alpha$, IL-6 and IL-1 $\beta$ in ischemic liver lobes were assessed by RT-qPCR. Consistent with a previous study (5), the results demonstrated that compared with those of the sham group the mRNA levels 
A

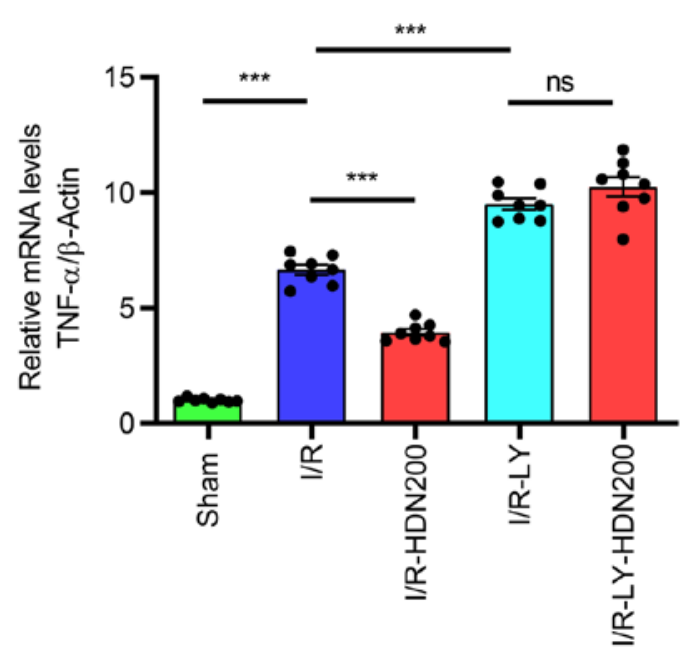

B

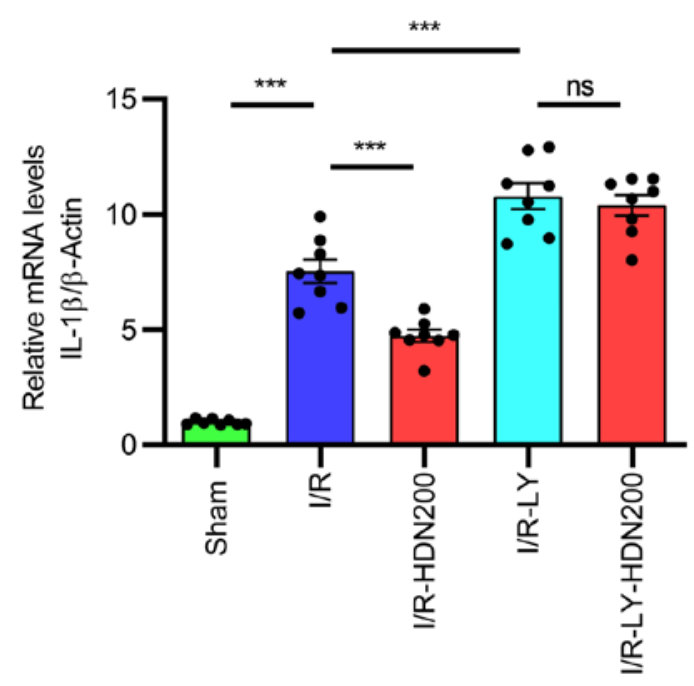

C

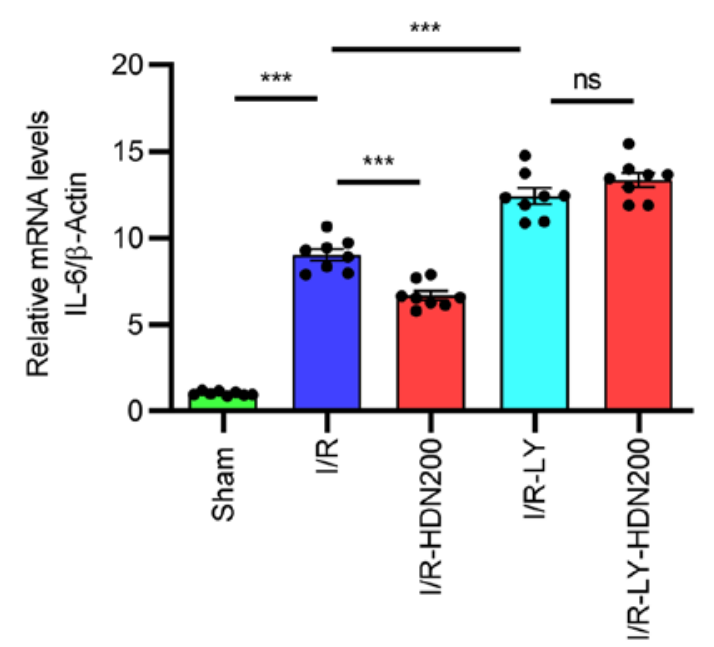

Figure 8. HDN suppresses liver inflammation during I/R injury through the Akt signaling pathway. mRNA expression levels of (A) TNF- $\alpha$, (B) IL-1 $\beta$ and (C) IL-6 assessed by reverse transcription-quantitative PCR in liver tissues from mice in sham, I/R, I/R with HDN treatment (200 mg/kg), I/R with Akt inhibitor (LY294002) treatment or I/R with LY294002 plus HDN treatment groups; $\beta$-Actin served as a control. $\mathrm{n}=8 \mathrm{mice} / \mathrm{group}$. Results are expressed as the relative fold increase from three experiments. ${ }^{* * *} \mathrm{P}<0.001$. HDN, hesperidin; I/R, ischemia/reperfusion; LY, LY294002; ns, not significant.

of TNF- $\alpha$, IL-6 and IL-1 $\beta$ in liver tissues increased significantly after I/R and HDN significantly attenuated these effects (Fig. 3A-C).

Hesperidin protects the liver against apoptosis following $I / R$ injury. Inhibition of apoptosis has been reported to have a modest protective effect on liver I/R injury (30). Thus, apoptotic markers in the livers of WT mice with or without HDN treatment after liver I/R injury was assessed. Western blotting results demonstrated that the expression levels of cleaved-caspase 3 increased markedly after liver I/R and were significantly reversed after HDN treatment (Fig. 4A). By contrast, no significant differences were observed in the levels of Bcl-2 in the liver after I/R compared with those of the sham group, but HDN treatment significantly increased the levels of Bcl-2 compared with those of the I/R group (Fig. 4A). In addition, TMR assay results demonstrated that there were significantly fewer TMR-positive apoptotic cells in livers in the I/R group treated with HDN compared with the number of apoptotic cells in the untreated I/R group (Fig. 4B).

Hesperidin activates the Akt pathway in liver I/R injury in vivo and in hepatocytes in vitro. Having determined that HDN ameliorates liver I/R injury, the mechanisms underlying the modulation of cellular functions by HDN were next examined. Akt serves a key role in protecting against liver I/R injury $(11,12)$, and several previous studies indicated that HDN induces Akt phosphorylation and influences apoptosis $(16,18,21,22)$. Thus, the relationship between HDN and the Akt pathway in liver I/R injury was investigated. No significant differences were observed in the levels of p-Akt in the liver after I/R compared with those of the sham group, but HDN increased the expression levels of p-Akt compared with those of the untreated I/R group (Fig. 5A). To ascertain the role of HDN in phosphorylating Akt in HCs, HCs were isolated and cultured in vitro (Fig. 5B). Cultured HCs were 
A

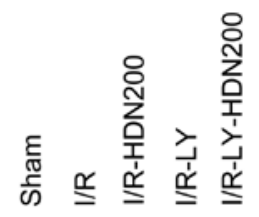

Pro-casp3

Cleaved-casp3

GAPDH

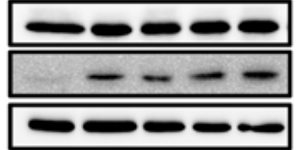

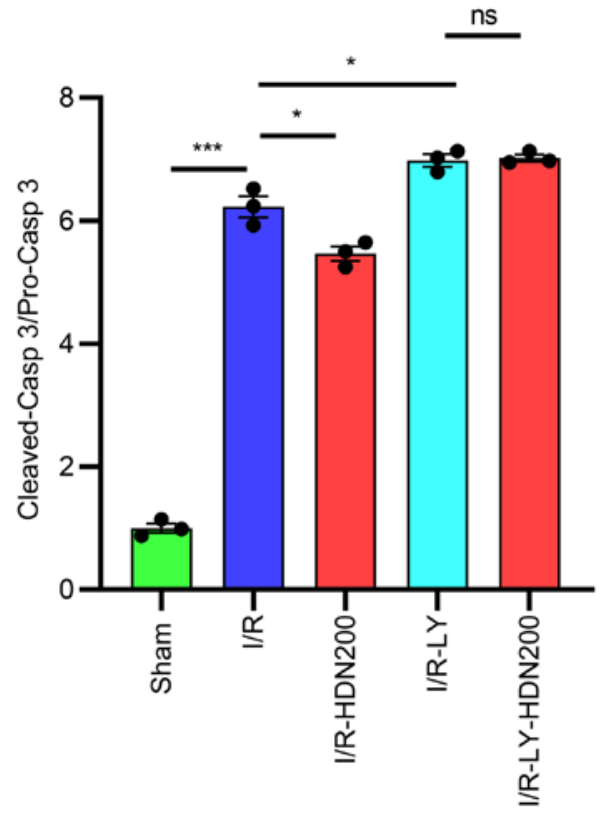

B

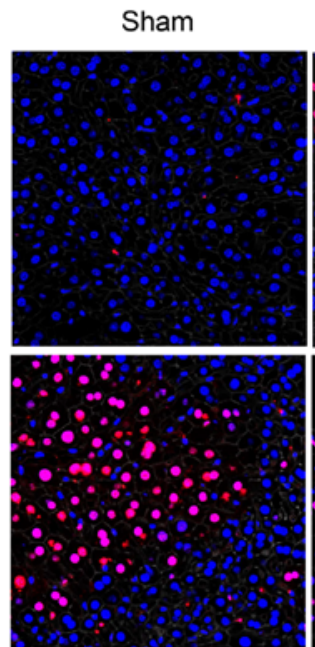

I/R-LY

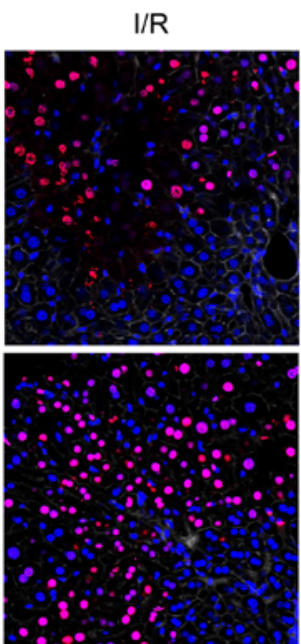

I/R-LY-HDN200

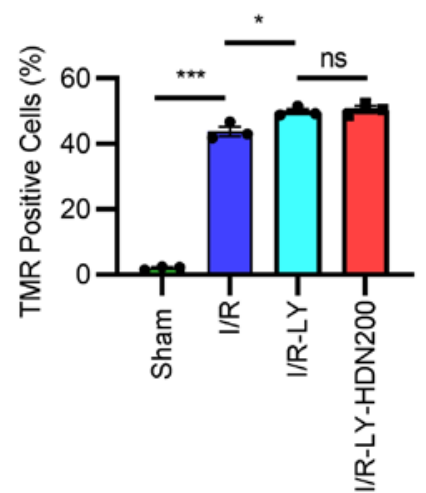

Figure 9. HDN protects the liver against apoptosis during I/R injury through the Akt signaling pathway. (A) Western blotting analysis of cleaved-casp3 in the liver of mice in the sham, I/R, I/R with HDN treatment $(200 \mathrm{mg} / \mathrm{kg}$ ), I/R with Akt inhibitor (LY294002) treatment or I/R with LY294002 plus HDN treatment. Each lane represents a separate animal. The blots shown are representative of three experiments with similar results. (B) Confocal images from mice liver stained with TMR red (red) and Hoechst nuclear stain (blue) after liver I/R injury with or without HDN treatment in the presence of LY294002. Magnification, $\mathrm{x} 40 . \mathrm{n}=8 \mathrm{mice} / \mathrm{group}$. Percentage of TMR-positive cells was quantified using ImageJ software and represented in a bar graph. ${ }^{*} \mathrm{P}<0.05,{ }^{* * * *} \mathrm{P}<0.001 . \mathrm{Casp} 3$, caspase 3; HDN, hesperidin; I/R, ischemia/reperfusion;LY: LY294002; ns; not significant.

exposed to HDN for $6 \mathrm{~h}$, and the results demonstrated that 10 and $20 \mathrm{ng} / \mathrm{ml} \mathrm{HDN}$ increased the levels of phosphorylated Akt in HCs (Fig. 5C).

Hesperidin ameliorates liver $I / R$ injury through the Akt pathway. To elucidate whether activation of the Akt pathway is necessary for HDN to ameliorate liver I/R injury, LY294002, which inhibits Akt activation and exacerbates liver injury after $\mathrm{I} / \mathrm{R}$, was used $(11,31)$. The results demonstrated that compared with those of the I/R group the expression levels of p-Akt were significantly decreased after administration of LY294002 (Fig. 6A). Notably, these effects were not rescued by the administration of HDN during liver I/R (Fig. 6A). In addition, consistent with the results of $\mathrm{p}$-Akt, the levels of serum ALT increased significantly after administration of
LY294002 compared with those of the I/R group (Fig. 6B). Furthermore, the protective effect of HDN was eliminated after administration of LY294002 (Fig. 6B). The elimination of the protective effect of HDN in the presence of LY294002 was also confirmed by the H\&E staining index (Fig. 6C).

Having determined that HDN ameliorated liver oxidative stress during liver I/R injury, whether the hepatoprotective effects were eliminated by the Akt inhibitor LY294002 was investigated. The results demonstrated that LY294002 significantly increased MDA levels and significantly decreased the levels of SOD, CAT, GPx and GSH compared with those of the respective I/R group, and these effects were unaffected by HDN in the presence of LY294002 (Fig. 7).

Having determined that the inflammatory response increased significantly after liver I/R and decreased markedly 
with HDN treatment, whether these effects were regulated by the Akt pathway was investigated. Cytokine expression levels after LY294002 administration were assessed; the results demonstrated that LY294002 significantly increased the mRNA expression levels of TNF- $\alpha$, IL- 6 and IL- $1 \beta$ compared with those of the untreated I/R group and that these effects were unaffected by co-treatment with HDN (Fig. 8).

It has been reported that the Akt inhibitor LY294002 promotes apoptosis during liver I/R $(12,32)$; thus, the effects of HDN and the Akt pathway in liver I/R were investigated. The results demonstrated that compared with those of the I/R group the levels of cleaved-caspase 3 significantly increased after treatment with LY294002 and that these effects were unaffected by HDN treatment in the presence of LY294002 (Fig. 9A). In addition, TMR assays demonstrated that there were a similar number of TMR-positive apoptotic cells in livers co-treated with LY294002 and HDN or LY294002 alone (Fig. 9B).

\section{Discussion}

HDN is a compound that is extracted from citrus fruits (13), that has been demonstrated to have potent pharmacological effects against oxidative stress, inflammation, microbes and cancer (13-16). However, the exact role and mechanisms of HDN in liver I/R injury remains unknown. The results of the present study demonstrated that HDN ameliorated liver I/R injury by decreasing liver oxidative stress, suppressing inflammatory responses and preventing hepatocyte apoptosis through the activation of the Akt pathway. Thus, HDN may be a useful factor for protection against liver injury. These findings raise the possibility that HDN may be used to ameliorate liver injury during $\mathrm{I} / \mathrm{R}$.

During the ischemia phase, blood flow is blocked and hepatocytes suffer from anaerobic metabolism and oxidative stress, which are considered to be the initiation of ischemic injury (9). Factors involved in oxidative stress, such as reactive oxygen species and nitric oxide, have been reported to have important roles in liver I/R injury (9). Oxygen radicals cause lipid peroxidation of cellular membranes, resulting in inflammatory cell infiltration, neutrophil activation and hepatic cell injury $(7-9,28)$. MDA, which is an oxidative damage indicator, is an essential product of lipid peroxidation (33). SOD, CAT, GPx and GSH, which can eliminate free radicals, are antioxidant indicators $(34,35)$. To determine the oxidative stress in liver I/R, the oxidative damage and antioxidant parameters were evaluated in the present study. The results demonstrated that MDA content increased markedly after liver I/R and was reversed by HDN treatment. The antioxidant indicators, SOD, CAT, GPx and GSH, decreased significantly after liver I/R; however, these levels were increased by HDN treatment. These data suggested that HDN may ameliorate liver oxidative stress during liver I/R injury and were consistent with previous studies (36-39). In a sepsis-induced lung injury model, Yuan et al (37) reported that oxidative stress was attenuated by HDN. In a sodium arsenite-induced nephrotoxicity and hepatotoxicity model, Turk et al (38) observed that HDN reduced oxidative stress and oxidative DNA damage depending on the dose. Similar results were observed in bilateral common carotid artery occlusion reperfusion injury (36) and in skeletal muscle I/R injury (39). However, how HDN ameliorates oxidative stress remains unknown.

Oxidative stress and nutrient deprivation initiate liver damage, which releases damage-associated molecular patterns (DAMPs), such as high mobility group box 1 and histone/DNA complexes (8). DAMPs induce inflammatory cell infiltration and activate innate immune responses, which leads to the release of pro-inflammatory cytokines and chemokines $(8,40,41)$. In the subsequent reperfusion phase, the inflammatory responses become more extensive and cause more inflammatory mediator release $(8,40,41)$. The pro-inflammatory milieu recruits and activates more innate and adaptive immune cells into the inflamed liver and causes more hepatocyte death $(8,40,41)$. Thus, the inflammatory response is both the result from and cause of liver damage. The results of the present study demonstrated that the mRNA expression levels of TNF- $\alpha$, IL- 6 and IL- $1 \beta$ in liver tissues increased significantly after I/R, and that HDN significantly attenuated these expression levels after liver I/R. These results suggested that HDN may attenuate inflammation in I/R. In a previous study, HDN was reported to reduce the secretion of inflammatory cytokines, differentiation and proliferation of cardiac fibroblasts by TGF- $\beta$ through the Notch 1 signaling pathway (42). In another infectious disease study, Ye et al (43) reported that HDN pretreatment significantly attenuated lipopolysaccharide-induced pulmonary injury and total protein concentration and markedly decreased the number of neutrophils and the levels of the inflammatory cytokines TNF- $\alpha$ and IL- 6 in an acute lung injury model in vivo and in vitro. Additionally, they also found that HDN binds directly with myeloid differentiation 2 (MD2) and inhibits MAPK activation, regulates I $\kappa \beta$ degradation, and blocks the interaction of MD2 and its co-receptor Toll-like receptor 4 TLR4 (43). Thus, HDN attenuates inflammation in liver I/R. However, the underlying mechanisms need to be further explored.

Several previous studies have indicated that apoptosis serves a key role in liver I/R $(5,30,44)$ and that inhibiting apoptosis attenuates liver injury (30). Additionally, it is reported that HDN ameliorates apoptosis in many situations (22,45-47). The results of the present study demonstrated that HDN suppressed apoptosis in liver $\mathrm{I} / \mathrm{R}$ injury, as indicated by increased $\mathrm{Bcl}-2$ and decreased cleaved-caspase 3 protein expression levels. In addition, HDN induced decreases in apoptotic cells were confirmed by TMR assays. Taken together, these data suggested that HDN suppressed apoptosis in liver I/R injury. These results corroborate with the effects of HDN in other I/R models. For example, Li et al (22) observed that short-term pretreatment with HDN protected against myocardial I/R injury by suppressing myocardial apoptosis. He et al (46) also observed that hesperetin (an active metabolite of HDN) post-treatment significantly inhibited apoptosis by increasing the expression of $\mathrm{Bcl}-2$ and decreasing the expression of Bax and cleaved-caspase 3, which diminished the apoptotic cardiomyocyte ratio in an in vitro cardiomyocyte model of hypoxia/reoxygenation injury. HDN also attenuated cerebral I/R injury through inhibiting the apoptotic pathway (47). However, studies have also reported that HDN induces apoptosis to reduce cancer cell prolif- 
eration $(15,48)$. These differences may be due to the acute nature of $\mathrm{I} / \mathrm{R}$ injury compared with the chronic induction of carcinoma.

The PI3K/Akt pathway is a protective pathway in liver I/R injury $(11,12,32,44,49)$. Akt is considered to be a potential therapeutic target for liver I/R (12). HDN activates the Akt pathway to protect cardiomyocytes in vivo and in vitro $(22,46,50)$. Consistent with these previous studies $(22,46,50)$, the present study demonstrated that HDN increased the levels of p-Akt after liver I/R injury and that treatment with the Akt inhibitor LY294002 diminished the protective effects of HDN in liver I/R injury. Thus, these results suggested that HDN supports Akt signaling to protect against liver I/R injury.

In conclusion, the present study revealed that HDN may protect against liver I/R injury by ameliorating oxidative stress, suppressing inflammatory responses and decreasing apoptosis through activating the Akt pathway. HDN is a potential therapeutic factor for treating liver I/R injury.

\section{Acknowledgements}

Not applicable.

\section{Funding}

This study was supported by The Young Teachers' Basic Ability Improvement in Guangxi University Project (grant no. 2019K Y0108), The National Natural Science Foundation of China (grant no. 81960358), Talents Sub-Highland of Emergency and Medical Rescue of Guangxi Province in China (grant no. GXJZ201405), Health Commission of Guangxi (grant no. Z2016289) and The Medical Excellence Award funded by the Creative Research Development Grant from the First Affiliated Hospital of Guangxi Medical University.

\section{Availability of data and materials}

The datasets used and /or analyzed during the current study are available from the corresponding authors on reasonable request.

\section{Authors' contributions}

SL designed and performed the majority of experiments, established the model mice, collected and analyzed the experimental data, and drafted the manuscript. QQ and DL performed the serum analysis and the H\&E staining. WP and YW isolated and cultured hepatocytes, and performed the immunofluorescence staining. YX performed the RT-qPCR and western blot analysis. JZ and LS supervised the project, provided technical advice, conducted analyses of the raw data, and reviewed and edited the manuscript. All authors read and approved the final manuscript.

\section{Ethics approval and consent to participate}

Animal protocols were approved by The Animal Care and Use Committee of The First Affiliated Hospital of Guangxi
Medical University (Nanjing, China), and the experiments were performed in adherence to the National Institutes of Health guidelines for the use of laboratory animals.

\section{Patient consent for publication}

Not applicable.

\section{Competing interests}

The authors declare that they have no competing interests.

\section{References}

1. Black CK, Termanini KM, Aguirre O, Hawksworth JS and Sosin M: Solid organ transplantation in the 21st century. Ann Transl Med 6: 409, 2018.

2. Czigany Z, Lurje I, Schmelzle M, Schöning W, Öllinger R, Raschzok N, Sauer IM, Tacke F, Strnad P, Trautwein C, et al: Ischemia-reperfusion injury in marginal liver grafts and the role of hypothermic machine perfusion: Molecular mechanisms and clinical implications. J Clin Med 9: 846, 2020.

3. Kadono K, Gruszynski M, Azari K and Kupiec-Weglinski JW: Vascularized composite allotransplantation versus solid organ transplantation: Innate-adaptive immune interphase. Curr Opin Organ Transplant 24: 714-720, 2019.

4. Nastos C, Kalimeris K, Papoutsidakis N, Tasoulis MK Lykoudis PM, Theodoraki K, Nastou D, Smyrniotis V and Arkadopoulos N: Global consequences of liver ischemia/reperfusion injury. Oxid Med Cell Longev 2014: 906965, 2014.

5. Sun P, Zhang P, Wang PX, Zhu LH, Du Y, Tian S, Zhu X and Li H: Mindin deficiency protects the liver against ischemia/reperfusion injury. J Hepatol 63: 1198-1211, 2015.

6. van Riel WG, van Golen RF, Reiniers MJ, Heger M and van Gulik TM: How much ischemia can the liver tolerate during resection? Hepatobiliary Surg Nutr 5: 58-71, 2016.

7. Peralta C, Jiménez-Castro MB and Gracia-Sancho J: Hepatic ischemia and reperfusion injury: Effects on the liver sinusoidal milieu. J Hepatol 59: 1094-1106, 2013.

8. Lu L, Zhou H, Ni M, Wang X, Busuttil R, Kupiec-Weglinski J and Zhai Y: Innate immune regulations and liver ischemia-reperfusion injury. Transplantation 100: 2601-2610, 2016.

9. Guan LY, Fu PY, Li PD, Li ZN, Liu HY, Xin MG and Li W: Mechanisms of hepatic ischemia-reperfusion injury and protective effects of nitric oxide. World J Gastrointest Surg 6: 122-128, 2014

10. Zhai Y, Petrowsky H, Hong JC, Busuttil RW and Kupiec-Weglinski JW: Ischaemia-reperfusion injury in liver transplantation--from bench to bedside. Nat Rev Gastroenterol Hepatol 10: 79-89, 2013.

11. Li S, Yi Z, Deng M, Scott MJ, Yang C, Li W, Lei Z, Santerre NM, Loughran P and Billiar TR: TSLP protects against liver I/R injury via activation of the PI3K/Akt pathway. JCI Insight 4: $129013,2019$.

12. Covington SM, Bauler LD and Toledo-Pereyra LH: Akt: A therapeutic target in hepatic ischemia-reperfusion injury. J Invest Surg 30: 47-55, 2017.

13. Parhiz H, Roohbakhsh A, Soltani F, Rezaee R and Iranshahi M: Antioxidant and anti-inflammatory properties of the citrus flavonoids hesperidin and hesperetin: An updated review of their molecular mechanisms and experimental models. Phytother Res 29: 323-331, 2015.

14. Elhelaly AE, AlBasher G, Alfarraj S, Almeer R, Bahbah EI, Fouda MMA, Bungău SG, Aleya L and Abdel-Daim MM: Protective effects of hesperidin and diosmin against acrylamide-induced liver, kidney, and brain oxidative damage in rats. Environ Sci Pollut Res Int 26: 35151-35162, 2019.

15. Pandey P, Sayyed U, Tiwari RK, Siddiqui MH, Pathak N and Bajpai P: Hesperidin induces ROS-mediated apoptosis along with cell cycle arrest at $\mathrm{G} 2 / \mathrm{M}$ phase in human gall bladder carcinoma. Nutr Cancer 71: 676-687, 2019.

16. Mo'men YS, Hussein RM and Kandeil MA: Involvement of $\mathrm{PI} 3 \mathrm{~K} / \mathrm{Akt}$ pathway in the protective effect of hesperidin against a chemically induced liver cancer in rats. J Biochem Mol Toxicol 33: e22305, 2019. 
17. Qin Z, Chen L, Liu M, Tan H and Zheng L: Hesperidin reduces adverse symptomatic intracerebral hemorrhage by promoting TGF- $\beta 1$ for treating ischemic stroke using tissue plasminogen activator. Neurol Sci 41: 139-147, 2020.

18. Qi W, Lin C, Fan K, Chen Z, Liu L, Feng X, Zhang H, Shao Y, Fang H, Zhao C, et al: Hesperidin inhibits synovial cell inflammation and macrophage polarization through suppression of the PI3K/AKT pathway in complete Freund's adjuvant-induced arthritis in mice. Chem Biol Interact 306: 19-28, 2019.

19. Selim NM, Elgazar AA, Abdel-Hamid NM, El-Magd MRA, Yasri A, Hefnawy HME and Sobeh M: Chrysophanol, physcion, hesperidin and curcumin modulate the gene expression of pro-inflammatory mediators induced by LPS in HepG2: In silico and molecular studies. Antioxidants 8: 371, 2019.

20. Jo SH, Kim ME, Cho JH, Lee Y, Lee J, Park YD and Lee JS: Hesperetin inhibits neuroinflammation on microglia by suppressing inflammatory cytokines and MAPK pathways. Arch Pharm Res 42: 695-703, 2019.

21. Justin-Thenmozhi A, Dhivya Bharathi M, Kiruthika R, Manivasagam T, Borah A and Essa MM: Attenuation of aluminum chloride-induced neuroinflammation and caspase activation through the AKT/GSK-3 $\beta$ pathway by hesperidin in wistar rats. Neurotox Res 34: 463-476, 2018.

22. Li X, Hu X, Wang J, Xu W, Yi C, Ma R and Jiang $\mathrm{H}$ : Short-term hesperidin pretreatment attenuates rat myocardial ischemia/reperfusion injury by inhibiting high mobility group box 1 protein expression via the PI3K/Akt pathway. Cell Physiol Biochem 39: 1850-1862, 2016.

23. Park HK, Kang SW and Park MS: Hesperidin ameliorates hepatic ischemia-reperfusion injury in Sprague-Dawley rats. Transplant Proc 51: 2828-2832, 2019.

24. Yazdani HO, Chen HW, Tohme S, Tai S, van der Windt DJ, Loughran P, Rosborough BR, Sud V, Beer-Stolz D, Turnquist HR, et al: IL-33 exacerbates liver sterile inflammation by amplifying neutrophil extracellular trap formation. $\mathrm{J}$ Hepatol 68: 130-139, 2017.

25. Lei Z, Deng M, Yi Z, Sun Q, Shapiro RA, Xu H, Li T, Loughran PA, Griepentrog JE, Huang H, et al: cGAS-mediated autophagy protects the liver from ischemia-reperfusion injury independently of STING. Am J Physiol Gastrointest Liver Physiol 314: G655-G667, 2018.

26. Sun Q, Loughran P, Shapiro R, Shrivastava IH, Antoine DJ, Li T, Yan Z, Fan J, Billiar TR and Scott MJ: Redox-dependent regulation of hepatocyte absent in melanoma 2 inflammasome activation in sterile liver injury in mice. Hepatology 65: 253-268, 2017.

27. Livak KJ and Schmittgen TD: Analysis of relative gene expression data using real-time quantitative PCR and the 2(-Delta Delta C(T)) method. Methods 25: 402-408, 2001.

28. Konishi T and Lentsch AB: Hepatic ischemia/reperfusion: Mechanisms of tissue injury, repair, and regeneration. Gene Expr 17: 277-287, 2017.

29. Abu-Amara M, Yang SY, Tapuria N, Fuller B, Davidson B and Seifalian A: Liver ischemia/reperfusion injury: Processes in inflammatory networks--a review. Liver Transpl 16: 1016-1032, 2010.

30. Bral M, Pawlick R, Marfil-Garza B, Dadheech N, Hefler J, Thiesen A and Shapiro AMJ: Pan-caspase inhibitor F573 mitigates liver ischemia reperfusion injury in a murine model. PLoS One 14: e0224567, 2019.

31. Li H, Chen O, Ye Z, Zhang R, Hu H, Zhang N, Huang J, Liu W and Sun X: Inhalation of high concentrations of hydrogen ameliorates liver ischemia/reperfusion injury through $\mathrm{A} 2 \mathrm{~A}$ receptor mediated PI3K-Akt pathway. Biochem Pharmacol 130: 83-92, 2017.

32. Zhang R, Zhang L, Manaenko A, Ye Z, Liu W and Sun X: Helium preconditioning protects mouse liver against ischemia and reperfusion injury through the PI3K/Akt pathway. J Hepatol 61: 1048-1055, 2014

33. Ayala A, Muñoz MF and Argüelles S: Lipid peroxidation: Production, metabolism, and signaling mechanisms of malondialdehyde and 4-hydroxy-2-nonenal. Oxid Med Cell Longev 2014: 360438, 2014.
34. Kurutas EB: The importance of antioxidants which play the role in cellular response against oxidative/nitrosative stress: Current state. Nutr J 15: 71, 2016.

35. Kuyumcu F and Aycan A: Evaluation of oxidative stress levels and antioxidant enzyme activities in burst fractures. Med Sci Monit 24: 225-234, 2018.

36. Praveen Kumar P, Sunil Kumar KT, Kavya Nainita M,Sai Tarun A, Raghu Ramudu BG, Deepika K, Pramoda A and Yasmeen C: Cerebroprotective potential of hesperidin nanoparticles against bilateral common carotid artery occlusion reperfusion injury in rats and in silico approaches. Neurotox Res 37: 264-274, 2020.

37. Yuan X, Zhu J, Kang Q, He X and Guo D: Protective effect of hesperidin against sepsis-induced lung injury by inducing the heat-stable protein 70 (Hsp70)/Toll-like receptor 4 (TLR4)/ Myeloid differentiation primary response 88 (MyD88) pathway. Med Sci Monit 25: 107-114, 2019.

38. Turk E, Kandemir FM, Yildirim S, Caglayan C, Kucukler S and Kuzu M: Protective effect of hesperidin on sodium arsenite-induced nephrotoxicity and hepatotoxicity in rats. Biol Trace Elem Res 189: 95-108, 2019.

39. Ekinci Akdemir FN, Gülçin I, Karagöz B, Soslu R and Alwasel SH: A comparative study on the antioxidant effects of hesperidin and ellagic acid against skeletal muscle ischemia/reperfusion injury. J Enzyme Inhib Med Chem 31 (sup4): 114-118, 2016.

40. Zhai Y, Busuttil RW and Kupiec-Weglinski JW: Liver ischemia and reperfusion injury: New insights into mechanisms of innate-adaptive immune-mediated tissue inflammation. Am J Transplant 11: 1563-1569, 2011.

41. Huang H, Tohme S, Al-Khafaji AB, Tai S, Loughran P, Chen L, Wang S, Kim J, Billiar T, Wang Y, et al: Damage-associated molecular pattern-activated neutrophil extracellular trap exacerbates sterile inflammatory liver injury. Hepatology 62: 600-614, 2015

42. Yu XH, Wang YF, Dai FY, Zhao JH and Li P: The protective effects of berberine and hesperidin on inflammatory factor-stimulating cardiac fibroblasts. Eur Rev Med Pharmacol Sci 23: 5468-5476, 2019.

43. Ye J, Guan M, Lu Y, Zhang D, Li C, Li Y and Zhou C: Protective effects of hesperidin on lipopolysaccharide-induced acute lung injury by targeting MD2. Eur J Pharmacol 852: 151-158, 2019.

44. Li Y, Tong L, Zhang J, Zhang Y and Zhang F: Galangin alleviates liver ischemia-reperfusion injury in a rat model by mediating the PI3K/AKT pathway. Cell Physiol Biochem 51: 1354-1363, 2018.

45. Hanchang W, Khamchan A, Wongmanee N and Seedadee C: Hesperidin ameliorates pancreatic $\beta$-cell dysfunction and apoptosis in streptozotocin-induced diabetic rat model. Life Sci 235: 116858, 2019.

46. He S, Wang X, Zhong Y, Tang L, Zhang Y, Ling Y, Tan Z, Yang $\mathrm{P}$ and Chen A: Hesperetin post-treatment prevents rat cardiomyocytes from hypoxia/reoxygenation injury in vitro via activating PI3K/Akt signaling pathway. Biomed Pharmacother 91: 1106-1112, 2017.

47. Wang JJ and Cui P: Neohesperidin attenuates cerebral ischemia-reperfusion injury via inhibiting the apoptotic pathway and activating the Akt/Nrf2/HO-1 pathway. J Asian Nat Prod Res 15: 1023-1037, 2013.

48. Naz H, Tarique M, Ahamad S, Alajmi MF, Hussain A, Rehman MT, Luqman S and Hassan MI: Hesperidin-CAMKIV interaction and its impact on cell proliferation and apoptosis in the human hepatic carcinoma and neuroblastoma cells. J Cell Biochem 120: 15119-15130, 2019.

49. Xiao Q, Ye Q, Wang W, Xiao J, Fu B, Xia Z, Zhang X, Liu Z and Zeng $X$ : Mild hypothermia pretreatment protects against liver ischemia reperfusion injury via the PI3K/AKT/FOXO3a pathway. Mol Med Rep 16: 7520-7526, 2017.

50. Li X, Hu X, Wang J, Xu W, Yi C, Ma R and Jiang H: Inhibition of autophagy via activation of $\mathrm{PI} 3 \mathrm{~K} / \mathrm{Akt} / \mathrm{mTOR}$ pathway contributes to the protection of hesperidin against myocardial ischemia/reperfusion injury. Int J Mol Med 42: 1917-1924, 2018.

This work is licensed under a Creative Commons Attribution-NonCommercial-NoDerivatives 4.0 International (CC BY-NC-ND 4.0) License. 